\title{
Chicas y chicos en una sociedad patriarcal innovadora
}

Andrea María Rodríguez. Universidad de Granada

Recepción: 27.07-2018 | Aceptado: 02.01.2019

Correspondencia a través de ORCID: Andrea María Rodríguez

Citar: Rodríguez Rivera, AM. (2019). Chicos y chicas en una sociedad patriarcal innovadora. REIDOCREA, 8,1-6. [ ]

\begin{abstract}
Resumen: El patriarcado continua presente en nuestra sociedad y en nuevos espacios como en el contexto del ciberabuso en el noviazgo de manera distintiva entre chicas/os. También, sobre la actitud positiva, actitud y percepción negativa que es percibida por la muestra de adolescentes. Este estudio plasma una exploración de dicho fenómeno. Objetivos: El propósito fundamental consistió en profundizar los roles que desempeñan las variables antecedentes tanto de la perpetración y victimización del ciberabuso como de la percepción social de un episodio de violencia de género a través del cibercontrol. Método: Investigación exploratoria realizada a una muestra de adolescentes de un Instituto de Secundaria Obligatoria. Chicas/os de $3^{\circ}$ y $4^{\circ}$ de Educación Secundaria respondieron a un cuadernillo de cuestionarios correspondientes a las variables estudiadas. Resultados: Se han encontrado algunas correlaciones significativas en el análisis estadístico con los resultados obtenidos. Conclusiones: Los mitos del amor romántico se relacionan con el apego ansioso en el ciberabuso y en las actitudes, manteniéndose la desigualdad con una resistencia brutal entre jóvenes en una etapa tan marcada e importante para la vida de una persona.
\end{abstract}

Palabras clave: Sexismo | Ciberabuso

\section{Girls and boys in an innovative patriarchal society}

\begin{abstract}
Patriarchy continues to be present in our society and in new spaces as in the context of cyber dating abuse in a distinctive way between girls and boys. Also in the positive attitude, negative attitude and perception that is perceived by the sample of adolescents. This study represents an exploration of this phenomenon. Objectives: The fundamental purpose was to study the roles played by the antecedent variables both in the perpetration and victimization of cyberbullying and in the social perception of a cybercontrol episode. Method: Exploratory research conducted on a sample of teenagers from Secondary School. Girls/boys of 3rd and 4th years of mandatory Secondary Education responded to a booklet of questionnaires corresponding to the variables studied. Results: Certain significant correlations were found in the statistical analysis with the results obtained. Conclusions: The myths of romantic love are related to anxious attachment in cyber dating abuse and in attitudes, maintaining inequality with a brutal resistance among young people in such a marked and important stage in the life of a person.
\end{abstract}

Keywords: Sexism | Cyberdating

\section{Introducción}

Actualmente vivimos en una sociedad patriarcal innovadora inmersa en una ideología machista que domina los comportamientos, pensamientos, sentimientos, roles...de chicas y chicos. Además, es creativa porque cada vez descubre nuevos espacios en la que desarrollarse afectando a las personas de determinada manera según su género, obstruyendo la libertad y la capacidad crítica por un arraigo de miles de años y que sigue transmitiéndose.

Aunque las Tecnologías de la Información y la Comunicación, TIC, presentan numerosas ventajas, ha conllevado consigo inconvenientes, especialmente para los jóvenes y menores, con el surgimiento de las nuevas formas de violencia mediante el uso de las tecnologías, como por ejemplo, el «sexting» -transmisión de imágenes de índole erótico sexual-, el «groo- ming»-acoso de forma sexual de un adulto hacía un 
menor- o el ciberacoso como forma de ejercer o sufrir violencia de género (Cabello, citado en Durán et al., 2015).

El ciberabuso en el noviazgo, Cyber Danting Abuse -CDA-, es aquel acoso, control, abuso y acecho por parte de la pareja mediante el empleo de las redes sociales y la tecnología (Zweig, Lachman, Yahner, y Dank, 2013). Según Borrajo, Gámez-Guadix y Calvete (2015), el control y la agresión directa son los dos tipos fundamentales de CDA. El control se asocia al empleo de las TIC para dominar a la pareja (dónde está, qué hace...), siendo un abuso más implícito. Mientras que la agresión directa se trata de comportamientos dirigidos a herir a la pareja (amenazar, insultar, divulgar información negativa sobre la persona...), siendo un abuso más explicito.

Por tanto, el interés sobre el CDA en esta investigación se centra en visualizar la problemática de la violencia de género en la adolescencia en este espacio y a la preocupación que surge en las personas que han sufrido la violencia de género en este contexto, tolerando conductas de control a través del móvil y las redes sociales.

\section{Objetivos o hipótesis}

El primer objetivo de dicha investigación se basó en considerar la relación de las variables de sexismo -total, hostil y benévolo-, mitos -total e idealización- y la dependencia -total, emocional, exclusiva y apego inseguro- con la percepción social de un episodio de violencia de género (VG) -actitud positiva y negativa-.

El segundo objetivo fue analizar en qué medida sexismo ambivalente (hostil y benévolo), mitos (total e idealización) y la dependencia (total, emocional, exclusiva y apego inseguro) son antecedentes de la victimización y de la perpetración del ciberabuso en el noviazgo (CDA), tanto en el componente de control como en el de agresión.

\section{Métodos}

\section{Participantes}

La muestra está formada por 167 estudiantes de $3^{\circ}$ y $4^{\circ}$ de educación secundaria obligatoria (ESO) con edades comprendidas entre 14 y 17 años. Entre los participantes hay 89 chicas y 75 chicos y una persona que no asigna su sexo. De los participantes el $53 \%$ ha tenido pareja alguna vez y el $27 \%$ tiene pareja en la actualidad.

\section{Instrumentos}

Sexismo. Se realizó un cuadernillo de cuestionarios empleando el Inventario de Sexismo Ambivalente para adolecentes compuesto por 20 ítems con la finalidad de medir el sexismo benévolo y el hostil, en una escala de respuesta que va desde el 1 (Muy en desacuerdo) a 6 (Muy de acuerdo), presentando una fiabilidad para sexismo benévolo de $\alpha=.77$ y sexismo hostil de $\alpha=.79$, siendo entre ambas de $\alpha=.84$. Mitos del amor romántico. La escala de mitos hacia el amor se conforma de 7 ítems con una fiabilidad de $\alpha=.58$ correspondiente al mito de idealización. Dependencia. La Spouse Specific Dependency Scale (SSDS) formada por 17 ítems que mide la dependencia exclusiva, emocional y el apego ansioso con una escala de respuesta de 6 puntos de tipo Likert en el que 1 se asocia a "Totalmente en desacuerdo" y 6 a "Totalmente de acuerdo". Episodio. Esta medida está constituida por la percepción negativa formada por 7 ítems con una escala de respuesta de 5 puntos de tipo Likert en el que 1 
corresponde a "Nada" y 5 a "Totalmente" haciendo referencia a la identificación de VG, gravedad del asunto, reconocimiento de quién tiene la responsabilidad de la reacción del chico perpetrador y sobre si este ejerce control sobre la chica. La actitud positiva (Pablo se siente responsable de Marta, está preocupado y la quiere) y la negativa (Pablo no confía en Marta, es egoísta y celoso) formada por 3 ítems cada una con una escala de respuesta de 7 puntos de tipo Likert en el que 1 corresponde a "Totalmente en desacuerdo" y 7 a "Totalmente de acuerdo". La percepción negativa presentó una fiabilidad de $\alpha=.62$, la actitud positiva de $\alpha=.52$ y la negativa de $\alpha=.57$. Abuso online en el noviazgo. Dicho cuestionario compuesto de 20 ítems en relación a las diversas tipologías de CDA, empleando la escala de respuesta de tipo Likert formada de 1 "Nunca" a 6 "Casi siempre". La fiabilidad de que una persona ha sido víctima de abuso es de $\alpha=.77$ y si la ha ejercido de $\alpha=.87$. Incluso se manifiestan conductas online de control y de agresión directa en la que la fiabilidad para control-víctima es de $\alpha=.77$ y de control-perpetrador es de $\alpha=.86$.

\section{Procedimiento}

Los cuestionarios se pasaron al alumnado de ocho grupos de ESO, cuatro grupos de $3^{\circ}$ ESO y cuatro de $4^{\circ}$ ESO el 8 de marzo de 2018 , en el salón de actos, con el propósito de que los participantes respondieran de manera más tranquila mientras reflexionaban.

Dicho cuadernillo incluía las medidas necesarias para su estudio, explicando las mismas instrucciones en cada uno de los grupos y garantizando la confidencialidad de los participantes durante la aplicación en la que se desarrolló en 30 minutos aproximadamente.

\section{Análisis de datos}

Los obtenidos de los instrumento usado son analizados a través de un análisis de correlaciones bivariadas y una regresión lineal múltiple.

\section{Resultados}

Los resultados del análisis de las muestras independientes de sexo (chicas y chicos) y de pareja (haber tenido o tener pareja, o ni haberla tenido ni tenerla) con la finalidad de contrastar medias y analizar las diferencias en relación a las variables estudiadas. En cuanto al sexo no se han encontrado disimilitudes significantes. Sin embargo, sí que se observaron diferencias significativas entre los estudiantes que habían tenido o tenían pareja y los que nunca la habían tenido, con respecto a la perpetración y victimización del ciberabuso online durante el noviazgo; los resultados se muestran a continuación en los siguientes gráficos 1 y 2.

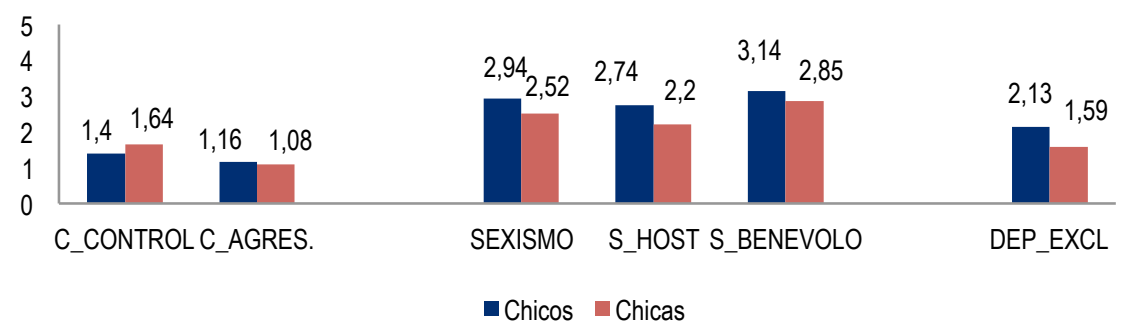

Gráfico 1. Diferencias por sexo 


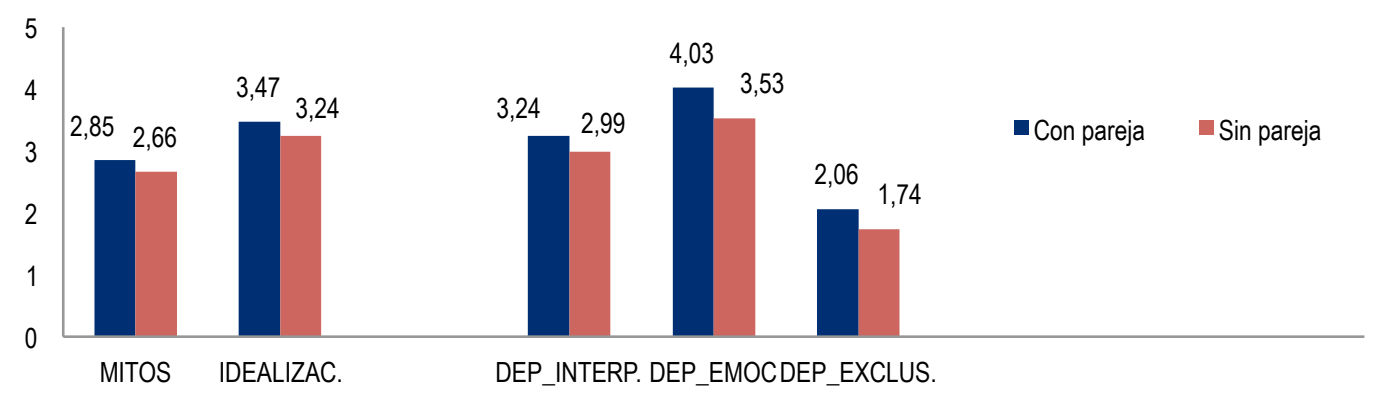

Gráfico 2. Diferencias haber tenido o tener pareja y nunca haberla tenido

Con respecto a los antecedentes de CDA en relación con la actitud positiva y actitud o percepción negativa, el análisis de correlaciones bivariadas muestra numerosas correlaciones significativas, tanto positivas como negativas, que se puede observar en las siguientes tablas 1 y 2 :

\begin{tabular}{|c|c|c|c|c|c|c|c|c|c|c|c|}
\hline \multirow[b]{2}{*}{ 1.Sexismo total } & 1 & 2 & 3 & 4 & 5 & 6 & 7 & 8 & 9 & 10 & 11 \\
\hline & & & & & & & & & & & \\
\hline 2.Sexismo hostil & $.83^{\star \star *}$ & & & & & & & & & & \\
\hline 3.Sexismo benévolo & $.90^{\star \star \star}$ & $.52^{\star \star \star}$ & & & & & & & & & \\
\hline 4. Mitos total & $.45^{\star \star *}$ & $.32^{\star \star *}$ & $.45^{\star \star \star}$ & & & & & & & & \\
\hline 5. Mitos idealización & $.40^{\star \star \star}$ & $.25^{\star \star *}$ & $.42^{\star \star \star}$ & $.96^{\star \star *}$ & & & & & & & \\
\hline 6. Dependencia total & $.57^{* \star *}$ & $.39^{* * *}$ & $.58^{\star \star *}$ & $.44^{\star \star *}$ & $.43^{\star * *}$ & & & & & & \\
\hline 7. Dependencia emocional & $.45^{\star \star *}$ & $.28^{\star * *}$ & $.48^{\star * \star}$ & $.45^{\star * \star}$ & $.46^{* \star \star}$ & $.88^{\star \star \star}$ & & & & & \\
\hline 8. Dependencia exclusiva & $.51^{* \star *}$ & $.41^{* * *}$ & $.48^{\star * \star}$ & $.20^{* *}$ & $.16^{*}$ & $.65^{\star * *}$ & $.44^{\star \star \star}$ & & & & \\
\hline 9. Apego ansioso & $.50^{* * *}$ & $.36^{* * *}$ & $.49^{\star * *}$ & $.34^{* * *}$ & $.33^{* * *}$ & $.80^{* * *}$ & $.54^{* \star *}$ & $.43^{\star * \star}$ & & & \\
\hline 10. Actitud positiva & $.23^{* *}$ & $.28^{* * *}$ & .14 & $.24^{* *}$ & $.18^{*}$ & $.24^{* *}$ & $.20^{*}$ & $.15^{\star}$ & $.20^{*}$ & & \\
\hline 11. Actitud negativa & $-.17^{\star}$ & $-.26^{\star \star \star}$ & -.06 & -.05 & .02 & .04 & .08 & -.13 & -.04 & .06 & \\
\hline 12. Percepción negativa & $-.28^{* * *}$ & $-.28^{\star \star *}$ & $-.22^{* *}$ & -.08 & .00 & -.05 & .04 & $-.18^{*}$ & -.11 & -.07 & $.54^{\star \star \star}$ \\
\hline
\end{tabular}

\begin{tabular}{|lcl|}
\hline & \multicolumn{2}{c|}{ Tabla 2. Tipo de actitud } \\
\hline Variables & Correlación & Tipo de actitud \\
\hline Sexismo hostil & $.23^{\star *}$ & Actitud positiva o tolerancia hacia el episodio de VG \\
& $-.28^{* * *}$ & Actitud negativa e intolerancia de la VG \\
\cline { 2 - 3 } Mitos del amor romántico & $.17^{*}$ & Actitud positiva o tolerancia hacia el episodio de VG \\
\hline${ }^{*} p<.05 ;{ }^{* *} p<.01 ;{ }^{* * *} p<.001$ & & \\
\hline
\end{tabular}

Con respecto al análisis de correlaciones bivariadas y el análisis exploratorio de las diferencias entre chicos y chicas en cuanto a la perpetración y victimización de CDA, se han encontrado correlaciones significativas tal como se expone en las tablas 3 y 4 :

\begin{tabular}{|c|c|c|c|c|c|c|c|c|c|c|c|c|c|c|c|}
\hline & & bla & Cor & acio & S & pe & tra & $n y$ & tim & iión & $C D$ & & & & \\
\hline & 1 & 2 & 3 & 4 & 5 & 6 & 7 & 8 & 9 & 10 & 11 & 12 & 13 & 14 & 15 \\
\hline 1.Sexismo total & & $.84^{* \star *}$ & $.93^{\star \star \star}$ & $.40^{* \star *}$ & $.38^{\star \star \star}$ & $.59^{\star \star \star}$ & $.51^{\star \star \star}$ & $.45^{\star \star \star}$ & $.53^{\star \star \star}$ & .24 & $.26^{*}$ & .06 & .15 & .10 & .23 \\
\hline 2.Sexismo hostil & $.81^{* * *}$ & & $.59^{\star \star *}$ & $.29^{\star *}$ & $.26^{*}$ & $.35^{\star \star}$ & $.28^{\star \star}$ & $.29^{\star *}$ & $.33^{\star *}$ & .16 & .18 & -.02 & .07 & .04 & .11 \\
\hline 3.Sexismo benévolo & $.88^{* * *}$ & $.42^{\star \star *}$ & & $.40^{\star * *}$ & $.39^{* * \star}$ & $.65^{\star * *}$ & $.57^{\star \star *}$ & $.49^{\star * *}$ & $.57^{\star \star *}$ & .25 & $.27^{*}$ & .10 & .18 & .12 & $.27^{*}$ \\
\hline 4.Mitos total & $.60^{\star \star \star}$ & $.44^{\star * *}$ & $.56^{* \star *}$ & & $.97^{\star \star \star}$ & $.49^{\star \star *}$ & $.55^{\star \star *}$ & $.27^{*}$ & $.34^{\star *}$ & .15 & .17 & -.05 & .09 & .04 & .10 \\
\hline 5.Mitos idealización & $.54^{* \star *}$ & $.37^{* *}$ & $.53^{\star * *}$ & $.95^{\star \star *}$ & & $.50^{\star * \star}$ & $.55^{\star \star *}$ & $.26^{*}$ & $.36^{\star *}$ & .13 & .17 & -.08 & .09 & .05 & .07 \\
\hline 6.Dependencia total & $.53^{\star \star *}$ & $.41^{\star \star *}$ & $.47^{\star \star \star}$ & $.40^{\star * *}$ & $.38^{* *}$ & & $.89^{* \star *}$ & $.64^{* * *}$ & $.83^{* \star *}$ & $.31^{*}$ & $.33^{* *}$ & -.04 & $.30^{*}$ & $.27^{*}$ & .22 \\
\hline 7.Dependen. emocional & $.38^{* *}$ & $.29^{*}$ & $.35^{\star \star}$ & $.34^{\star *}$ & $.36^{\star *}$ & $.87^{\star * \star}$ & & $.46^{\star \star \star}$ & $.57^{\star \star \star}$ & .23 & $.26^{*}$ & -.05 & .22 & .20 & .15 \\
\hline 8.Dependen. exclusiva & $.48^{\star \star *}$ & $.37^{\star *}$ & $.43^{\star * *}$ & .20 & .16 & $.69^{\star \star *}$ & $.45^{\star \star *}$ & & $.43^{* \star *}$ & .10 & .11 & -.03 & .07 & .04 & .15 \\
\hline 9.Apego ansioso & $.44^{\star \star \star}$ & $.37^{\star *}$ & $.37^{\star *}$ & $.37^{* *}$ & $.34^{* *}$ & $.74^{\star * *}$ & $.48^{\star * *}$ & $.42^{\star * *}$ & & $.36^{* *}$ & $.37^{\star *}$ & .03 & $.36^{* *}$ & $.32^{*}$ & $.26^{*}$ \\
\hline 10.Ciberyo total & .11 & .14 & .05 & .21 & .23 & .22 & .25 & -.10 & .28 & & $.97^{\star * *}$ & $.45^{\star * *}$ & $.82^{* \star *}$ & $.80^{* * \star}$ & $.47^{* * *}$ \\
\hline 11.Ciberyo control & .07 & .12 & .00 & .19 & .24 & .24 & .27 & -.11 & .28 & $.92^{\star * *}$ & & $.30^{*}$ & $.78^{* * *}$ & $.80^{* * *}$ & $.34^{* *}$ \\
\hline 12.Ciberyo agresión & .05 & .09 & .00 & .17 & .11 & .10 & .12 & -.11 & .20 & $.76^{\star \star *}$ & $.48^{* *}$ & & $.45^{\star \star *}$ & $.30^{*}$ & $.74^{* * *}$ \\
\hline 13.Ciberpareja total & .00 & .03 & -.03 & .10 & .08 & .10 & .13 & -.19 & .21 & $.78^{\star \star *}$ & $.64^{* * *}$ & $.72^{\star * \star}$ & & $.96^{\star \star *}$ & $.61^{* * *}$ \\
\hline 14.Ciberpareja control & -.11 & -.04 & -.14 & -.01 & -.03 & .01 & .06 & -.26 & .13 & $.75^{\star \star *}$ & $.68^{* \star *}$ & $.59^{\star * \star}$ & $.94^{\star * *}$ & & $.39^{* *}$ \\
\hline 15.Ciberpareja agresión & .13 & .12 & .08 & .24 & .19 & .14 & .11 & -.04 & .23 & $.62^{\star * *}$ & $.37^{\star}$ & $.81^{* \star *}$ & $.77^{\star \star *}$ & $.55^{\star \star *}$ & \\
\hline
\end{tabular}




\begin{tabular}{|llcl|}
\hline \multicolumn{4}{c|}{ Tabla 4. Resultados según el sexo } \\
\hline Sexo & Variables predictoras & Correlaciones & Variables dependientes \\
\hline Chicas & Apego ansioso & $.44^{* *}$ & Perpetración en general \\
& Apego ansioso & $.45^{* *}$ & Ciber-control \\
& Apego ansioso & $.39^{* *}$ & Victimización en general \\
& Apego ansioso & $.38^{* *}$ & Ciber-control \\
\cline { 2 - 4 } Chicos & Sexismo hostil & $.37^{*}$ & Perpetración en general \\
& Dependencia emocional & $.39^{*}$ & Ciber-control \\
\hline${ }^{*} p<.05 ;{ }^{* *} p<.01 ;{ }^{* * *} p<.001$ & & \\
\hline
\end{tabular}

Finalmente se realiza el análisis de regresión lineal múltiple jerarquizada por pasos, el cual muestra diferencias significativas por el sexo; concretamente para chicas la variable criterio fue el apego ansioso y la variable predictora fue el sexismo benévolo $(\beta=.52, p=.00)$; sin embargo para los chicos la variable criterio fueron el sexismo hostil y la dependencia emocional, y las variables predictoras fueron para ambas los mitos del amor romántico (respectivamente: $\beta=.37, p=.01 ; \beta=.31, p=.04$ ). En la tabla 4 se muestran los resultados con más detalle.

\begin{tabular}{|lll|}
\hline \multicolumn{3}{|c|}{ Tabla 4. Resultados de la regresión lineal por pasos } \\
\hline Sexo & Variable criterio & Variable predictora \\
\hline Chicas & Apego ansioso & Sexismo benévolo $(\beta=.52, p=.00)$ \\
\cline { 2 - 3 } Chicos & Sexismo hostil & Mitos del amor romántico $(\beta=.37, p=.01)$ \\
& Dependencia emocional & Mitos del amor romántico $(\beta=.31, p=.04)$ \\
\hline
\end{tabular}

\section{Discusión}

Los resultados obtenidos apuntan a que existen discrepancias entre chicas y chicos en cuanto a la perpetración y victimización de CDA, pues las chicos son más perpetradores de ciber-agresión y las chicas de comportamientos de ciber-control. También destaca que el sexismo hostil sigue siendo el principal predictor de actitudes de justificación de comportamientos abusivos en la pareja en este nuevo contexto. Sin embargo, se ha hallado la asociación negativa entre la no justificación del episodio de cibercontrol y el sexismo benévolo. La ideología sexista benévola puede favorecer el que se considere que las chicas deben ser cuidadas y protegidas por sus parejas, y que perciban las conductas de control como muestras de amor y preocupación por parte de la pareja más que como formas de controlarlas (Moya et alt., 2007). Además, el sexismo benévolo se ha manifestado como única predictora en las chicas con respecto al apego ansioso.

También se comprueba que el apego ansioso es predictor de la victimización y perpetración de CDA en general y de ciber-control, en cuanto a las chicas; Tal como comenta Monje (2016), con respecto a la victimización, las personas que son dependientes emocionales presentan una elevada ansiedad ante la pérdida de la pareja o por el miedo a la soledad. Ésta, afecta a ambos sexos, predominando más en la mujer, convirtiéndose en perpetradora debido a su baja autoestima y autoconcepto negativo por la asignación de los problemas que se puedan generar en la relación de pareja.

En cuanto a los chicos, el sexismo hostil, en el caso de la perpetración de CDA, y la dependencia emocional para la predicción de la perpetración de ciber-control son las dos variables de mayor peso predictivo. Esto evidenciaría la relevancia de la ideología machista en el contexto de las nuevas tecnologías al igual que en los estudios de violencia de género tradicional, donde se ha relacionado, en el caso de los hombres, con una mayor probabilidad de ejercer violencia sobre su pareja y la mayor 
culpabilización de la mujer por la violencia sufrida (Garaigordobil y Aliri, 2013). Además, resalta la creencia en los mitos del amor romántico como principal antecedente del sexismo hostil. Dicho resultado va en la línea de los estudios que halla en la creencia de los mitos del amor romántico, la facilidad del establecimiento de relaciones basadas en la sumisión de la chica y la dependencia emocional (De la Peña et al., 2011). Además, la dependencia emocional en los chicos tiene de nuevo como principal predictor la creencia en los mitos del amor romántico. Tal y como exponen Míngez y Álvarez (2013), los mitos se encuentran asociados con relaciones dependientes y con ideas incoherentes sobre el amor. Por ello, quizás se sigan manteniendo el mito de la media naranja, de la omnipotencia, de la compatibilidad amor-violencia para permanecer en la relación; así como el mito de los celos como una manifestación de la dependencia emocional.

Este estudio ha tenido limitaciones, la primera que no es posible considerar la relación causa-efecto entre las variables estudiadas por tratarse de una investigación de naturaleza correlacional. La necesidad de una muestra más numerosa y representativa de la población adolescente es otra de las limitaciones ya que aunque la muestra del estudio es considerada amplia, le resta validez y limita la generalización de los resultados.

A pesar de las limitaciones los resultados demuestran la necesidad de diseñar propuestas de intervención que favorezcan la no dependencia emocional hacia la pareja tanto en chicas como en chicos. Los resultados de dicha investigación, avalan la importancia de combatir los mitos del amor romántico y la ideología sexista, tanto hostil como benévola. En España encontramos algunas propuestas promovidas por el Instituto de la Mujer, como el "Programa de prevención de la violencia en las relaciones de noviazgo de jóvenes y adolescentes" elaborado por Muñoz et al., (2010) que abordan dichos contenidos pero no intervienen en las relaciones de dependencia emocional.

\section{Referencias}

Borrajo, E., Gámez-Guadix, M., \& Calvete, E. (2015). Justification beliefs of violence, myths about love and cyber dating abuse. Psicothema, 27(4), 327-333.

De la Peña, EM., Ramos, E., Luzón, JM. y Recio, P. (2011). Andalucía Detecta: sexismo y violencia de género en la juventud. Instituto Andaluz de la Mujer. Consejería para la Igualdad y Bienestar Social. Junta de Andalucía.

Durán, M., y Martínez-Pecino, R. (2015). Ciberacoso mediante teléfono móvil e Internet en las relaciones de noviazgo entre jóvenes. Comunicar, 44, 159-167.

Garaigordobil, M. y Aliri, J. (2013). Relaciones del sexismo con justificación de la violencia, y con otras formas de prejuicio como la dominancia social y el autoritarismo. Estudios de Psicología, 34 (2), 127-139.

Mínguez, L. y Álvarez, L. (2013). Estilo de apego y estilo de amar. Trabajo Fin de Grado de Enfermería. Cantabria: Universidad de Cantabria.
Monje, N. (15.07.2016). Dependencia emocional: cuando el amor no tiene límites. Psicología Serendipia [Blog]. [Archived by WebCite ${ }^{\circledR}$ at http://www.webcitation.org/755bK70mR]

Moya, M., Glick, P., Expósito, F., De Lemus, S., \& Hart, J. (2007). It's for Your Own Good: Benevolent Sexism and Women's Reactions to Protectively Justified Restrictions. Personality and Social Psychology Bulletin, 33, 1421-1434.

Muñoz, MJ., González, P., Fernández, L., Sebastián, J., Peña, ME. y Perol, O. (2010). Validación de un programa de prevención de la violencia en las relaciones de noviazgo de jóvenes y adolescentes. Madrid: Instituto de la Mujer.

Zweig, J., Lachman, P., Yahner, J., \& Dank, M. (2013). Correlates of Cyber Dating. Journal of Youth and Adolescence, 43(8), 1306-1321. 